\title{
Valley-Hall Topological Transport in Graphene Plasmonic Crystal Waveguides
}

\author{
Jian Wei You ${ }^{1}$, Yupei Wang ${ }^{1}$, and Nicolae. C. Panoiu ${ }^{1}$ \\ ${ }^{1}$ University College London, Department of Electronic and Electrical Engineering, Torrington Place, WC1E 7JE, \\ London, United Kingdom. e-mail: n.panoiu@ucl.ac.uk
}

\begin{abstract}
Due to immunity to disorder and structural imperfections, topologically-protected plasmonic modes have recently attracted increasing attention. Here, we introduce two different mechanisms to construct valley-Hall domain-wall interface waveguides in graphene plasmonic crystal to mimic the quantum valley-Hall effect. In the first case, we break the in-plane spatial inversion symmetry of a single-layer graphene plasmonic crystal waveguide to achieve valleyHall topological characteristics, whereas in the second case, we break the out-of-plane spatial inversion symmetry of a bi-layer graphene plasmonic crystal waveguide to implement the analog quantum valley-Hall effect. A molecular sensor based on this valley-Hall topological transport phenomenon is also be presented.
\end{abstract}

\section{INTRODUCTION}

By emulating the quantum Hall effect (QHE), quantum valley-Hall effect (QVHE) and quantum spin-Hall effect (QSHE) observed in solid-state physics, topologically protected edge modes have been achieved in both linear and nonlinear photonic systems [1-4]. Recently, several studies revealed that topologically protected onewave edge plasmons can be realized by breaking the time reversal symmetry to mimic the QHE in graphene metasurfaces [5-7]. In this work, we show that the topological transport of plasmons in graphene plasmonic crystal waveguides can also be realized in a time reversal symmetric manner by exploiting the idea of valleycontrast transport.

\section{QUANTUM VALLEY-HALL EFFECT IN SINGLE-LAYER GRAPHENE PHOTONIC CRYSTAL WAVEGUIDE}

The designed single-layer graphene photonic crystal waveguide (GPCW) [8] is illustrated in Fig. 1(a). A domain-wall interface highlighted by a transparent green strip is constructed by putting together two graphene nanohole crystals referred to as domains D1 and D2. The domain D1 is the mirror-symmetric counterpart of the domain D2, with respect to a plane passing through the domain-wall interface and perpendicular onto the y-axis. As a consequence, the K-valley points of the D1 domain correspond to the K'-valley points of the D2 domain. Since the chirality of the K-valley is opposite to that of the K'-valley, a chirality-momentum locked edge mode will emerge in the topological bandgap of the single-layer GPCW.

In order to verify this idea, the projected band diagram of this single-layer GPCW is evaluated through fullwave calculations, and the result is depicted in Fig. 1(b), where a valley-Hall topological edge-mode band (blue line) is observed inside the topological bandgap. To further confirm the topologically-protected unidirectional propagation feature of the waveguide modes, a straight GPCW is studied and the results are presented in Fig. 1(c). In the simulation, a source located at the middle of the GPCW, as illustrated by the inset of Fig. 1(c) where six dipoles with $\pi / 3$ phase difference have been marked by red spots, generates right-circularly polarized (RCP) monochromatic light with frequency $v_{0}$. As a result of the valley-chirality-locking feature, unidirectional propagation of plasmonic waves is achieved. In addition to the unidirectional propagation, the topological feature of backscattering-immune propagation in the proposed GPCW is also validated in Fig. 1(d). A monochromatic light is launched at the waveguide port " 1 ", whereas the transmitted power is collected at the waveguide port " 2 ". The near-field distribution in Fig. 1(d) shows the light propagation is immune to the $\Omega$-sharp bend. 
In practical applications, the intrinsic loss of graphene is critical in the design of GPCW. Thus, we have investigated the effect of graphene loss on the characteristic of the valley-Hall topological plasmon propagation in the single-layer GPCW, and the results have been summarized in Fig. 1(e). It shows the optical power of the waveguide mode, which evaluated via a surface integral of the Poynting vector over a cross section in the $y-z$ plane, decreases as the propagation distance increases. To further investigate the influence of graphene loss, the electric field distributions at lifetime $\tau=5 \mathrm{ps}$ and $\tau=1$ ps have been evaluated, and the results have been presented in Fig. 1(f) and Fig. 1(g), respectively. These results illustrated clearly the dramatic effect that the intrinsic graphene loss has on the characteristic propagation length of the topological waveguide modes.
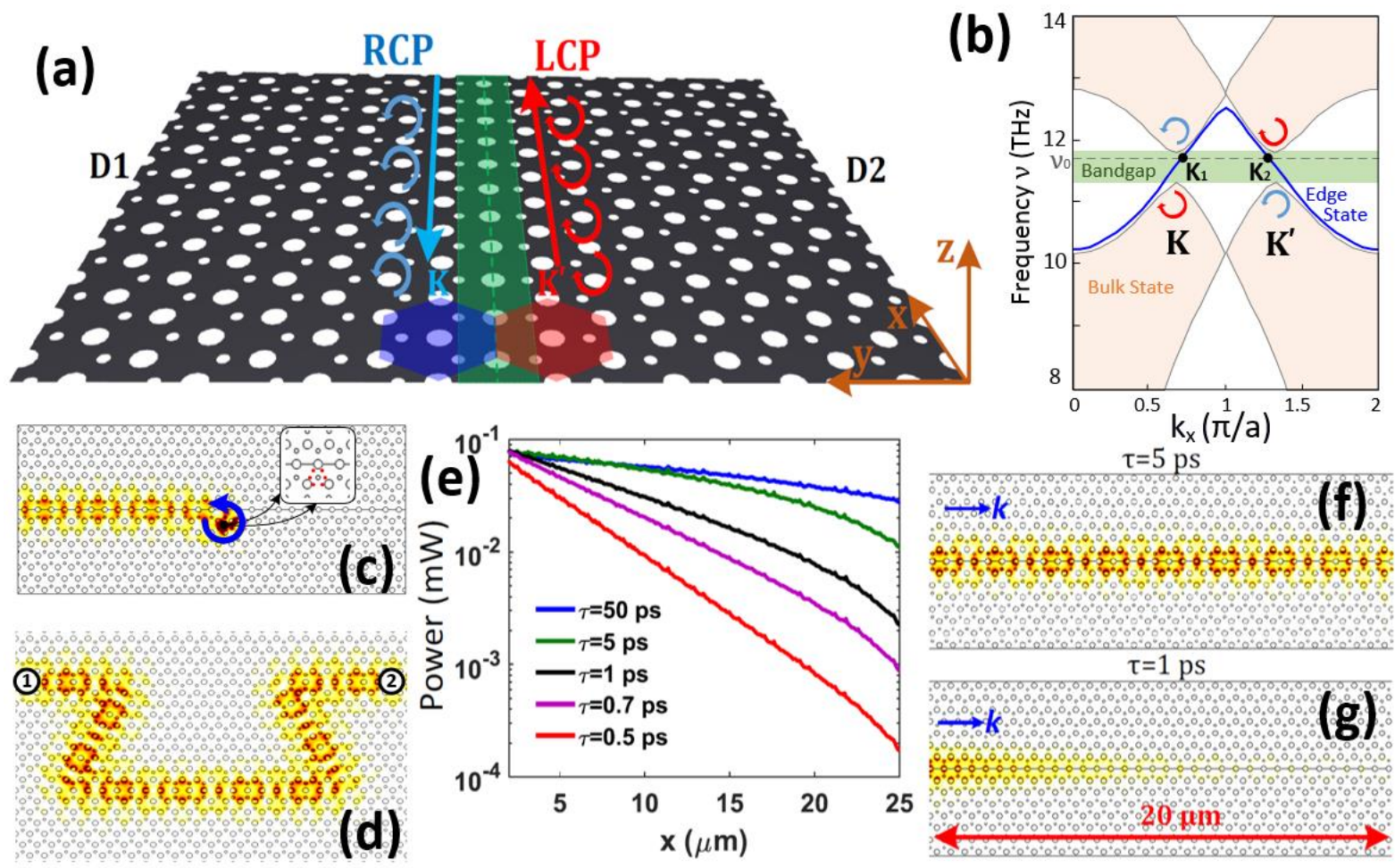

Fig. 1. (a) Schematic of a topological valley-Hall waveguide supporting valley-contrast unidirectional transport of terahertz plasmonic modes. (b) Projection of the band diagram of the graphene nanohole plasmonic crystal along the $\Gamma$-K direction. (c) Unidirectional propagation along $-x$ axis is achieved upon excitation with RCP light source. (d) Defect-immune propagation. (e) Dependence of the power on the propagation distance determined for different lifetimes of the graphene plasmon. Field distribution $|\mathrm{E}|$ of a terahertz plasmon propagating in a lossy graphene nanohole plasmonic crystal waveguide, determined for graphene carrier lifetime of (f) 5 ps and (g) 1 ps.

\section{QUANTUM VALLEY-HALL EFFECT IN BILAYER GRAPHENE PHOTONIC CRYSTAL WAVEGUIDE}

The proposed bi-layer GPCW [9] is illustrated in Fig. 2(a). It consists of two freestanding, optically coupled graphene plasmonic crystals with the same unit cell. However, the left- and right-hand side domains of the top graphene layer are horizontally shifted, in opposite directions, by a certain distance to break the out-of-plane spatial inversion symmetry of the top and bottom GPCWs. When the shift distance is zero, the unit cell of this bilayer GPCW belongs to $\mathrm{D}_{6 \mathrm{~h}}$ point symmetry group, thus it possesses six Dirac cones. However, when a non-zero shift distance is introduced, the mirror symmetry between the top and bottom graphene layers is broken. As a result, the $\mathrm{D}_{6 \mathrm{~h}}$-symmetry-protected Dirac cones are gapped out, and a frequency band gap emerges.

In order to construct a domain-wall interface that can possess topological interface modes, we put together two bilayer graphene metasurfaces with a non-zero shift distance in a mirror-symmetric manner, as depicted in Fig. 2(a). The projected band diagram of this finite bilayer GPCW consisting of 20 unit cells along the y-axis and periodic along the $\mathrm{x}$-axis is evaluated, and the results are given in Fig. 2(b). The topological interface modes are marked by red lines, the green regions represent the bulk states and the blue lines indicate edge modes confined 
at the metasurface boundaries. In order to gain deeper physical insights into the properties of these interface and edge modes, the field distribution of the interface mode "1" in Fig. 2(b) is given in Fig. 2(c), where the electric field is strongly confined at the domain-wall interface. The field distribution of the edge mode " 2 " in Fig. 2(b) is given in Fig. 2(d), where the electric field is now confined at the boundary of the GPCW.
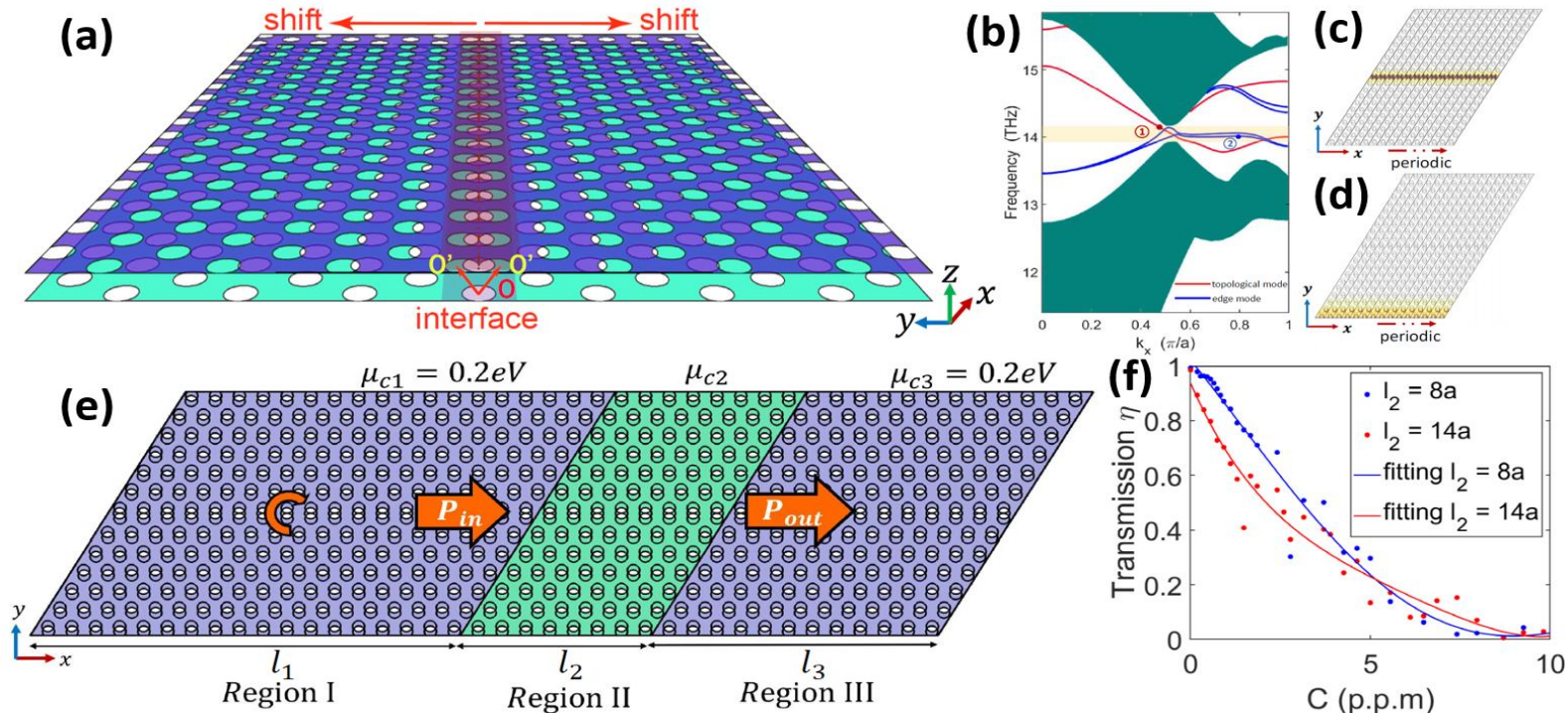

Fig. 2. (a) Schematic of the bilayer graphene metasurface. (b) Projected band diagram (green region), topological interface modes (red lines), and non-topological edge modes (blue lines), determined for a finite bilayer graphene metasurface with width of 20 unit cells and $\mathrm{s}=100 \mathrm{~nm}$. (c), (d) Field distributions of a topological interface mode and edge mode, marked by 1 and 2 in panel (b), respectively. (e) Schematic of the molecular sensor. The topological interfacial mode carries an input and output power in the regions I and III, respectively. An additional bilayer metasurface in region II is used to detect the concentration of adsorbed $\mathrm{NO}_{2}$ molecules. (f) Light transmission vs. $\mathrm{C}_{\mathrm{NO} 2}$ of $\mathrm{NO} 2$ gas, determined for $l_{2}=8 a$ and $l_{2}=14 a$.

Due to the tunable chemical potential of graphene and the unidirectional propagation feature of the topological interface mode of the bi-layer GPCW, a highly sensitive molecular sensor has been designed, and its schematic is depicted in Fig. 2(e). This sensor consists of three bilayer GPCWs, marked as regions I, II, and III. The gas molecules can be adsorbed only in the region II, thus the chemical potential in the region II varies with the concentration of gas molecules, which is a particular type of chemical doping. Consequently, the transmission of this molecular sensor is dependent on the gas concentration, as shown in Fig. 2(f). It shows the transmission decreases steeply when the gas concentration increases, and this gas sensor can be used to detect the gas variations in a broad range of molecular concentrations.

\section{REFERENCES}

[1] T. Ozawa, et al., "Topological photonics," Rev. Mod. Phys., vol. 91, no. 1, p. 015006, 2019.

[2] L. Lu, J. D. Joannopoulos, and M. Soljacic, "Topological photonics," Nat. Photonics, vol. 8, no. 11, p. 821-829, 2014.

[3] S. Kruk, et al., "Nonlinear light generation in topological nanostructures," Nat. Nanotechnol., vol.14, p. 126-130, 2019.

[4] Z. Lan, J. W. You, N. C. Panoiu, "Nonlinear one-way edge-mode interactions for frequency mixing in topological photonic crystals," Phys. Rev. B, vol.101, no. 15, p. 155422, 2020.

[5] D. Jin, T. Christensen, M. Soljacic, N. X. Fang, L. Lu, X. Zhang, "Infrared topological plasmons in graphene," Phys. Rev. Lett., vol. 118, p. 245301, 2017.

[6] D. Pan, R. Yu, H. Xu, F. J. Garcia de Abajo, "Topologically protected Dirac plasmons in a graphene superlattice," Nat. Commun., vol. 8, p. 1243, 2017.

[7] J. W. You, Z. Lan, N. C. Panoiu, "Four-wave mixing of topological edge plasmons in graphene metasurfaces," Sci. Adv., vol. 6, no. 13, p. eaaz3910, 2020.

[8] J. W. You, Z. Lan, Qiaoliang Bao, N. C. Panoiu, "Valley-Hall topological plasmons in a graphene nanohole plasmonic crystal waveguide," IEEE J. Sel. Top. Quantum Electron., (to be published, DOI: 10.1109/JSTQE.2020.2982991).

[9] Y. Wang, J. W. You, Z. Lan, N. C. Panoiu, "Topological valley plasmon transport in bilayer graphene metasurfaces for sensing applications," Opt. Lett. (to be published). 